\title{
Emmanuelle Labeau et Pierre Larrivée (dir.), Les temps du passé français et leur enseignement
}

Chiara Preite

\section{CpenEdition}

\section{Journals}

Édition électronique

URL : https://journals.openedition.org/studifrancesi/39981

DOI : 10.4000/studifrancesi.39981

ISSN : 2427-5856

Éditeur

Rosenberg \& Sellier

Édition imprimée

Date de publication : 1 décembre 2004

Pagination : 427-429

ISSN : 0039-2944

\section{Référence électronique}

Chiara Preite, «Emmanuelle Labeau et Pierre Larrivée (dir.), Les temps du passé français et leur enseignement ", Studi Francesi [En ligne], 143 (XLVIII | II) | 2004, mis en ligne le 30 novembre 2015, consulté le 19 mai 2021. URL : http://journals.openedition.org/studifrancesi/39981 ; DOI : https:// doi.org/10.4000/studifrancesi.39981

Ce document a été généré automatiquement le 19 mai 2021.

\section{cc) (†) $\ominus$}

Studi Francesi è distribuita con Licenza Creative Commons Attribuzione - Non commerciale - Non opere derivate 4.0 Internazionale. 


\title{
Emmanuelle Labeau et Pierre Larrivée (dir.), Les temps du passé français et leur enseignement
}

\author{
Chiara Preite
}

\section{RÉFÉRENCE}

EMMANUELLE LABEAU et PIERRE LARRIVÉE (dir.), Les temps du passé français et leur enseignement, Cahiers Chronos, n 9, Rodopi B.V., Amsterdam-New York, NY 2002, 241 pp.

1 Les contributions réunies dans cet ouvrage invitent à la réflexion critique sur les descriptions des temps du passé du système verbal français et sur leur enseignement sous un point de vue synchronique, évolutif ou contrastif.

Dulcie M. ENGEL, Les nouveaux temps du passé?, pp. 1-13. Dans le but de tracer un aperçu général des formes verbales qu'on appelle «nouveaux temps du passé» en français par rapport au passé simple (PS), Engel présente le système du passé, passant en revue le passé composé $(\mathrm{PC})$, le présent narratif (PR), le plus-que-parfait $(\mathrm{PQP})$ et le passé surcomposé (PSC). Forme analytique et premier des temps nouveaux en compétition avec le PS, le PC ne peut pas remplacer le PS parce qu'ils expriment les faits du passé de deux différents points de vue (introduction et conclusion de l'histoire vs corps du récit). La richesse sémantique du PR dépend de sa portée panchronique, due à l'absence de tout morphème temporel. Il ne peut toutefois remplacer un PS «plus épique et héroïque» que si un contexte temporel a été établi auparavant. Le PQP non-antérieur semble être de plus en plus utilisé à l'oral, où le PS recule, pour distinguer les faits qui ont une certaine distance temporelle par rapport au présent. Cependant, son emploi n'est pas si répandu que celui du PR. Enfin, le PSC est le plus récent et le plus rare des nouveaux temps du passé: il a eu origine dans une variation régionale, mais il n'est pas en concurrence avec les autres. En conclusion de son excursus, Engel affirme que les 
effets particuliers crées par la combinaisons des temps sont plus remarquables que l'emploi d'une forme spécifique.

3 Douglas L. RIDEOUT, L'opposition perfectif / imperfectif dans le passé français, pp. 15-29. Rideout avance l'hypothèse que l'opposition perfectif/imperfectif dans le système aspectuel et temporel du passé français s'explique par le rapport entre la structure temporelle interne d'une situation et un point de référence a; ce dernier se situant à l'extérieur de la situation présentée comme un tout dans le cas du perfectif et à l'intérieur dans le cas de l'imperfectif. Après une présentation de la difficulté provoquée par cette opposition aux apprenants du FLE (amenés par les grammaires à l'envisager en termes de différence temporelle et non'aspectuelle), l'auteur précise la structure interne d'une situation (la borne A marque le commencement de la situation représentée par un verbe et $\mathrm{B}$ sa fin; a est le point d'où le locuteur contemple la situation), pour arriver ensuite à l'examen des caractéristiques de l'imperfectivité (imparfait) et de la perfectivité (passé simple et passé composé à valeur prétérit) décrites par de nombreux grammairiens. Rideout conclut en proposant la validation, par les effets textuels, du rapport d'interaction entre l'aspect grammatical, le sens du verbe et sa structure temporelle interne: la présence d'une forme perfective indique une transition, tandis qu'une forme imperfective marque une continuation.

Marie-Eve RITZ, The semantics of the passé composé in contemporary French: towards a unified representation, pp. 31-50. En opposition avec la grammaire traditionnelle qui attribue plusieurs valeurs au passé composé (PC), Ritz avance une vision unifiée et homogène de ce temps verbal. Après un examen des caractéristiques sémantiques du PC, des théories grammaticales et des problèmes causés par sa description sémantique, l'auteur analyse le PC ayant recours aux théories concernant le temps et l'aspect, dans le but d'en proposer une «représentation sémantique». Selon Ritz, afin de développer la signification des propositions contenant le PC, il est nécessaire de prendre en considération la valeur du temps verbal même, la fonction temporelle spécifique de certains adverbes qui l'accompagnent et qui fixent le point de référence à l'aide duquel il est possible d'envisager l'action (ce que le PC ne peut pas faire seul, à la différence du passé simple), la catégorie aspectuelle et le rapport interactionnel entre les phrases du discours.

Pierre LARRIVÉE, Sémantique conceptuelle et sémantique référentielle du passé composé, pp. 51-69.

6 Selon Larrivée le passé composé (PC) montre bien la complexité du sens propre des langues naturelles grâce à la division du sens en plusieurs niveaux de représentation en interaction. Chaque unité linguistique est associée à une valeur conceptuelle qui la caractérise et cette dernière permet, par ses interactions avec le sens des autres éléments du contexte, de fixer la valeur référentielle que l'unité linguistique y acquiert. En explorant certains des paramètres qui auraient pu conduire à la définition du PC, tels que la perfectivité (représentation d'un événement aboutissant à un terme envisagé dans son extériorité) et la terminativité, le linguiste a remarqué qu'ils ne s'appliquent pas à la valeur conceptuelle du verbe, mais seulement à sa valeur référentielle. En effet, le PC semble ne pas se définir en termes de borne finale d'un événement: les inférences terminatives ne résultent pas de la valeur intrinsèque de la forme verbale, mais d'un effet dérivé de la structuration dénotative des situations, par rapport à notre connaissance du monde. Par conséquent, le PC ne saurait être analysé 
comme représentant perfectif du passé, ne possédant pas de valeur terminative intrinsèque.

7 Françoise LABELLE, Point de vue et aspect en français et en anglais, pp. 71-89. Sur la base des travaux de C.Smith sur le découpage aspectuel, F.Labelle analyse les formes verbales du passé en français et en anglais, dans la tentative d'expliquer certaines erreurs d'emploi du passé composé et de l'imparfait que font les anglophones en français L2, et ceux que commettent les francophones en anglais L2. S'appuyant sur une série d'exemples, l'auteur affirme que la conception binaire de l'aspect (perfectif/imperfectif) doit être élargie à la catégorie du «neutre» qui serait compatible avec un emploi ouvert ou fermé: si l'on exclut le passé simple, les formes simples du français sont neutres. Le simple past (contrairement au passé composé et au passé simple) peut présenter les procès d'état comme ouverts tout en étant un perfectif, parce qu'il nous positionne dans le passé au moment où l'événement se déroulait. Parfois il est rendu en français par l'imparfait (imperfectif) parce que ce dernier étant neutre, il peut même présenter une situation fermée. Labelle essaie également de résoudre le "casse-tête» du present perfect: partant de l'hypothèse de $\mathrm{W}$. Klein qui rejette l'incompatibilité entre ce temps et les adverbes de temps temporellement définis en anglais, l'auteur constate qu'en français la portée du présent est plus vaste, probablement à cause de son aspect neutre, mais elle conclut sur la nécessité de réflexions ultérieures.

Arie MoLENDIJK, La structuration logico-temporelle du texte: le passé simple et l'imparfait du français, pp. 91-104. Après une analyse des oppositions traditionnelles concernant le passé simple (PS) et l'imparfait (IMP) (perfectif / imperfectif, premier plan / arrière plan, ponctuel / duratif, postériorité / simultanéité), A.Molendijk montre qu'il est possible d'examiner la différence entre PS et IMP en termes purement temporels, ce qui aiderait les locuteurs non-francophones à éviter certains emplois erronés. L'auteur montre ensuite que le choix de l'un ou de l'autre temps dans un passage déterminé du texte dépend du type de rapport logique qui peut être établi entre deux phrases. La question est donc de savoir à quel type de rapport logique on a affaire: cela dépend du «contenu temporel» du PS qui exprime la postériorité, l'inclusion temporelle ou l'antériorité par rapport au moment de la parole, et de l'IMP qui n'exprime que la simultanéité globale, liée étroitement à la notion de "présupposition temporelle».

Bénédicte FACQUES, Passé composé, imparfait et présent dans les récits journalistiques: des alternances aux ruptures temporelles, pp. 105-133. Envisagée comme un phénomène relevant de la cohésion verbale et renvoyant à tout changement d'un temps à un autre temps dans la syntagmatique des textes, l'«alternance temporelle» est analysée sur la base de l'alternance passé composé (PC) / imparfait (IMP) dans un corpus de récits journalistiques. B.Facques examine d'abord les fonctions de l'alternance PC/IMP dans les récits journalistiques, qui ne se réduit pas aux relations prédicatives (succession et simultanéité) instaurées par les temps du récit historique et fictionnel (passé simple et imparfait). À cause de leur hétérogénéité temporelle, aspectuelle et énonciative, PC et IMP forment un couple moins cohésif que l'alternance PS/IMP, mais participant de la cohérence des textes journalistiques. Ensuite, les alternances temporelles sont analysées dans le cadre particulier du discours rapporté et de la polyphonie afin de cerner leur rôle lorsqu'elles accompagnent les évaluations et les explications de l'auteur, les énoncés métadiscursifs et la modalisation idéologique. Seules les alternances temporelles à fonction modalisatrice sont considérées comme des ruptures dans l'organisation énonciative des récits journalistiques parce qu'elles coupent le récit 
pour introduire le marquage idéologique du locuteur et, par conséquent, sa prise de distance à l'égard des voix narratives.

Anne JUDGE, Écarts entre manuels et réalités: un problème pour l'enseignant des temps du passé à des étudiants d'un niveau avancé, pp. 135-156. La fréquence des fautes à l'écrit dans l'emploi des temps du passé de l'indicatif des étudiants anglophones d'un niveau avancé aussi bien que des étudiants Erasmus et des Français mêmes, a amené A.Judge à formuler l'hypothèse selon laquelle les étudiants seraient déroutés par le fait que les manuels scolaires ne tiennent pas assez compte de l'évolution de l'emploi des temps du passé à l'écrit. Après avoir encadré conceptuellement les problèmes posés par l'imparfait (IMP), le passé composé (PC), le passé simple (PS) et le présent narratif (PrN), l'auteur en examine le traitement de la part des manuels consultés, afin d'illustrer leurs représentations des concepts liés aux systèmes du récit et du discours ainsi qu'au système du PrN. Si les manuels sont assez compétents en ce qui concerne le traitement des différences entre PC et PS dans les systèmes récit/discours et entre PC/ PS et IMP, ils n'enseignent pas aux étudiants à reconnaître le nouveau système multifocal (système n'ayant aucun temps-pivot stable) et sa richesse de connotations. Deux dernières remarques concernent l'IMP, dont certains emplois semblent aller contre la règle et déroutent ainsi les étudiants, et le PrN, fréquemment utilisé par les étudiants même sans encouragement à cause de sa simplicité morphologique aussi bien que de sa flexibilité du point de vue du registre.

11 Emmanulle LABEAU, Circonstants atténuants?: L'adjonction de localisateurs temporels aux formes passées dans la production écrite d'apprenants anglophones avancés, pp. 157-179. Selon E. Labeau la difficulté de la part des allophones dans l'usage des tiroirs du passé est due aux affinités qui existent entre certains circonstants temporels et les temps du passé. Les tiroirs du passé composé (PC) et de l'imparfait (IMP) analysés ici peuvent se combiner avec les mêmes indicateurs temporels; de plus la combinaison d'un circonstant et d'un tiroir n'a pas toujours la même acceptabilité, ce qui rend très complexe le choix entre eux. L'auteur décrit les facteurs qui influencent le choix du PC et de l'IMP afin de corriger la sursimplification des descriptions pédagogiques qui amènent à la production d'énoncés fautifs. Plusieurs remarques découlent de l'étude du corpus qui méritent d'être systématisées dans le but d'éclaircir la discrimination entre PC et IMP à l'usage des anglophones. Entre autres:l'IMP est incompatible avec les verbes téliques; les verbes statiques se combinent avec l'IMP pour indiquer un état mais avec le PC pour présenter un événement; l'IMP est incompatible avec un intervalle précis, une durée limitée ou une limite initiale, etc. Des règles d'emploi tenant compte de ces remarques devraient être proposées dans des termes accessibles aux apprenants allophones non spécialisés en linguistique.

12 Martin HowARD, L'acquisition des temps du passé en français par l'apprenant dit avancé: une approche lexicale, pp. 181-204. Cette étude examine les rapports entre l'apprentissage de la morphologie verbale employée pour exprimer la temporalité du passé et l'acquisition du lexique verbal de la langue cible (LC) chez l'apprenant avancé. Il en résulte un rôle déterminant du lexique dans l'acquisition de la grammaire de la LC, les deux ne pouvant être séparés qu'artificiellement. M.Howard analyse l'acquisition du passé composé (PC) et de l'imparfait (IMP) d'un point de vue lexical: la valeur d'un lexème verbal est l'un des facteurs qui influencent la répartition des temps du passé. En effet, si le locuteur natif est capable de conjuguer tout verbe au PC comme à l'IMP pour aboutir à des effets de sens très spécifiques, l'apprenant a plutôt tendance à conjuguer certains 
verbes à un certain temps prototypique. Un autre facteur de discrimination est le rôle du milieu d'acquisition par rapport au développement de la morphologie temporelle et du lexique verbal. Il apparaît que la production de la morphologie des temps du passé ne pose pas de problèmes importants à l'apprenant avancé, et que la variation fait partie de l'emploi productif de la morphologie temporelle des étudiants. Elle se manifeste à travers le suremploi de certaines formes à l'IMP, l'emploi restreint de l'IMP à différentes formes verbales par rapport au PC et l'emploi peu fréquent de verbes conjugués aux deux temps: la répartition inégale des formes verbales à chaque temps reflète l'importance de la valeur lexicale du verbe pour la morphologisation temporelle.

Urszula Paprocka-PiotrowsKa, 'Mais dans ce moment le chien est venu' ou comment les apprenants formels polonophones s'approprient le système temporel du français, pp. 205-229. Cette réflexion, issue d'une étude plus large sur le développement de la compétence linguistique des apprenants polonophones, est un bilan de l'apprentissage de la transmission de l'information temporelle des informateurs au $6^{\mathrm{e}}$ et au $12^{\mathrm{e}}$ mois d'acquisition. Divisée en deux volets, cette communication d'une part présente les informateurs, les données (production de récits de la part des étudiants examinés) et le cadre d'analyse adopté (exploitation de moyens directs et indirects pour rendre compte des dimensions temporelles); d'autre part elle présente les résultats des analyses pour chaque groupe. Les conclusions qui en découlent permettent de dégager quelques tendances générales concernant l'acquisition du temps et de l'aspect en français par les locuteurs polonophones. Entre autres, l'auteur affirme l'utilisation jusqu'au $12^{\mathrm{e}}$ mois d'acquisition, du présent grammatical en tant que temps de base "par défaut» et la présence, dès les stades élémentaires, de la morphologie verbale, qui toutefois ne devient fiable que plus tard et dont la stabilisation ne va pas de pair avec le recours conscient aux oppositions aspectuelles. 